\title{
Aplicativo sobre a detecção precoce do autismo: uma ferramenta educacional para o ensino em saúde
}

Application on the early detection of autism: an educational tool for teaching in health

Aplicación sobre la detección precoz del autismo: una herramienta educativa para la enseñanza en salud

Karina Saunders Montenegro ${ }^{1 *}$, Marcelo Alberto Barbosa Figueiredo ${ }^{2}$, Leny Silene de Freitas Castro ${ }^{1}$. Kátia Simone Kietzer ${ }^{1}$.

\section{RESUMO}

Objetivo: Demonstrar o desenvolvimento de um aplicativo sobre detecção precoce do autismo à luz das Diretrizes para Atenção à Reabilitação do Transtorno do Espectro Autista (TEA), descritas pelo Ministério da Saúde, para dar subsídios ao ensino de acadêmicos e profissionais de saúde e suas práticas educativas. Métodos: $O$ aplicativo foi desenvolvido por meio de um trabalho interdisciplinar entre um terapeuta ocupacional, que forneceu o referencial teórico para o desenvolvimento do aplicativo, e um profissional da área de processamento de dados que programou o software. O sistema foi desenvolvido utilizando a ferramenta App Inventor. Resultados: $O$ aplicativo foi construído com sucesso. Um aplicativo que permite receber, armazenar e analisar dados referentes aos indicadores precoces do autismo. Todos os sinais de alerta para o TEA foram organizados por faixa etária de zero a seis meses, seis a 12 meses, 12 a 18 meses, 18 a 24 meses e 24 a 36 meses. Para cada faixa etária foram descritos um conjunto de comportamentos. Conclusão: $O$ aplicativo atua como instrumento de apoio no ensino em deteç̧ão precoce do autismo. Sua utilização possibilita a agilidade nos processos de tomada de decisão, no âmbito do ensino e dos serviços de saúde.

Palavras-chave: Software, Educação Em Saúde, Transtorno Autístico, Diagnóstico Precoce.

\section{ABSTRACT}

Objective: To demonstrate the development of an application on the early detection of autism in the light of the Guidelines for Attention to Rehabilitation of Autistic Spectrum Disorder (TEA), described by the Ministry of Health, to give subsidies to the teaching of academics and health professionals and their practices education. Methods: The application was developed through an interdisciplinary work between an occupational therapist, who provided the theoretical framework for application development, and a professional in the data processing area who programmed the software. The system was developed using the App tool Inventor. Results: The application was successfully built. An application that allows to receive, store and analyze data related to the early indicators of autism. All of the warning signs for ASD were organized by age group of zero to six months, six to 12 months, 12 to 18 months, 18 to 24 months and 24 to 36 months. A set of behaviors was described for each age group. Conclusion: The application acts as a support tool in teaching early detection of autism. Its use enables agility in the decision-making processes, within the scope of teaching and health services.

Key words: Software, Education and Health, Autistic Disorder, Early Diagnosis.

1Universidade do estado do Pará (UEPA), Belém-PA. *E-mal: karinasmonte@yahoo.com.br

Empresa de Tecnologia da Informação e Comunicação do Estado do Pará (PRODEPA), Belém-PA.

SUBMETIDO EM: $12 / 2018$

ACEITO EM: $12 / 2019$

PUBLICADO EM: $2 / 2019$ 


\section{RESUMEN}

Objetivo: Demostrar el desarrollo de una aplicación sobre detección precoz del autismo a la luz de las Directrices para la Atención a la Rehabilitación del Trastorno del Espectro Autista (TEA), descritas por el Ministerio de Salud, para dar subsidios a la enseñanza de académicos y profesionales de salud y sus prácticas educativo. Métodos: La aplicación fue desarrollada por medio de un trabajo interdisciplinario entre un terapeuta ocupacional, que proporcionó el referencial teórico para el desarrollo de la aplicación, y un profesional del área de procesamiento de datos que programó el software.El sistema fue desarrollado utilizando la herramienta App inventor. Resultados: La aplicación se ha construido con éxito. Una aplicación que permite recibir, almacenar y analizar datos referentes a los indicadores precoces del autismo. Todas las señales de alerta para el TEA se organizaron por grupo de edad de cero a seis meses, de seis a doce meses, de 12 a 18 meses, de 18 a 24 meses y de 24 a 36 meses. Para cada grupo de edad se describieron un conjunto de comportamientos. Conclusión: La aplicación actúa como instrumento de apoyo en la enseñanza en detección precoz del autismo. Su utilización posibilita la agilidad en los procesos de toma de decisión, en el ámbito de la enseñanza y de los servicios de salud.

Palabras clave: Software, Educación En Salud, Trastorno Autístico, Diagnóstico Precoz.

\section{INTRODUÇÃO}

Falar sobre o ensino inovador na formação de futuros profissionais da área da saúde é romper com um ensino baseado apenas na multicausalidade de doenças, na intervenção e no olhar fragmentado do sujeito. É necessário incentivar e promover uma aprendizagem voltada para a integralidade do indivíduo, com foco em um ensino com responsabilidade social, voltado para o aprendizado centrado nas inter-relações entre o aluno e professor e o meio onde está inserido, desenvolvendo uma prática transformadora da realidade.

Para um ensino inovador em saúde é fundamental o rompimento com as metodologias de ensino tradicionais, e o aumento dos incentivos a favor da construção de currículos integrados, com foco na interdisciplinaridade e orientado pela competência (BARBA et al., 2012).

As estratégias tradicionais de ensino representam uma abordagem conteudista, compartimentada e organizada em disciplinas. O ensino tradicional esteve, por muito tempo, centrado na ação do professor, como agente ativo do processo de ensino e aprendizagem, que determinava como e o que deveria ser aprendido pelos alunos (CAMPOS et al., 2009; TEMPSK e BORBA, 2009).

Em contrapartida as metodologias ativas, visam evitar a relação de dependência, estabelecida tradicionalmente entre aluno e professor, promovendo um ensino inovador, centrado no aluno, no qual o mesmo torna-se corresponsável por seu aprendizado, participando ativamente da construção do conhecimento.

Para uma formação inovadora o discente deve ser preparado para desenvolver não apenas um conjunto de ideias, conhecimentos e habilidades, como, também, construir um arcabouço de interesses, atitudes, disposições e padrões de comportamento (BARBA et al., 2012).

A partir do conceito de ensino inovador destaca-se a aprendizagem móvel, que esta sendo cada vez mais utilizada e que traz consigo contribuições para o processo de ensino e aprendizagem em saúde, como custo acessível, facilidade no transporte, por serem portáteis, fácil manuseio e multitarefas. Os aplicativos para dispositivos móveis corroboram com o conceito de aprendizagem móvel e são considerados como ferramentas educacionais inovadoras que favorecem a comunicação, informação e educação de acadêmicos da saúde (DE OLIVEIRA e ALENCAR, 2017). Porém, apesar destes benefícios, observa-se a carência de ferramentas educacionais inovadoras, que auxiliem o ensino de acadêmicos de saúde durante as aulas 
práticas, garantindo um material adequado de acordo com o que é preconizado pelo Ministério da Saúde, e que garanta ao mesmo tempo rapidez e agilidade ao serviço, em virtude da grande demanda do SUS.

O uso de aplicativos móveis são novas fontes de informação utilizadas com muita frequência e que possuem um grande potencial para serem utilizadas no meio acadêmico, representando uma inovação no processo de ensino aprendizagem, em especial na área da saúde (DE OLIVEIRA e ALENCAR, 2017).

É notório que a saúde pública no Brasil passa por desafios relacionados a grande demanda populacional a ser assistida, o que torna emergencial a busca por soluções para intervir neste problema, a computação móvel apresenta um grande potencial em suprir estas necessidades, oferecendo benefícios como a melhora da qualidade dos serviços prestados à população, redução de custos e da demanda reprimida ( BARROS et al, 2011).

Os estudos de Dos Santos et al. (2017) corroboram ao afirmar que a criação e o uso de aplicativos em saúde contribuem nos processos de prevenção em saúde, visto que na assistência clínica prevalece uma carência de testes e protocolos, tão comuns em pesquisas científicas, o que representa a necessidade de mais estudos com o uso deste tipo de ferramenta.

Refletindo sobre o desafio de formar profissionais de saúde que atuem na integralidade da atenção, voltados para a promoção e prevenção da saúde, e em virtude do aumento expressivo do número de casos e da realização do diagnóstico tardio do transtorno do espectro do autismo (TEA) no Brasil, torna-se necessário o desenvolvimento de ferramentas acadêmicas que auxiliem profissionais e acadêmicos de saúde a realizarem a detecção precoce de sinais de alerta para o TEA.

O TEA é um transtorno neurobiológico considerado um dos que mais geram prejuízos no desenvolvimento infantil (ALCKMIN-CARVALHO et al., 2013; ALCKMIN-CARVALHO et al., 2014; NYGREN et al., 2011; SCHWARTZMAN e ARAÚJO, 2011).

O diagnóstico deste transtorno é de responsabilidade de equipes multidisciplinares que atuam nos cuidados primários de saúde, sendo fundamental a detecção dos sinais de alerta precocemente ainda no primeiro ano de vida. Infelizmente no Brasil, o diagnóstico é tardio, por volta dos seis e sete anos de idade (ALCKMIN-CARVALHO et al., 2014; NYGREN et al., 2011; OZONOFF et al., 2010 ). Estudos apontam que os profissionais de saúde não estão preparados para realizarem a detecção precoce do TEA durante o acompanhamento do desenvolvimento infantil (FLORES e SMEHA, 2013).

Assim, buscando contribuir com as práticas educativas de acadêmicos de saúde, realizadas em programas de vigilância do desenvolvimento infantil, foi realizada a produção de uma ferramenta tecnológica (aplicativo), para ser utilizada em aparelhos celulares e tablets, como ferramenta de auxílio ao estudo e detecção precoce dos sinais de alerta para o TEA.

O aplicativo apresenta formato em check list com sinais de alerta para o TEA, organizado por faixa etária de 0 a 36 meses de idade, de acordo com as orientações das Diretrizes de Atenção à Reabilitação da Pessoa com Transtornos do Espectro do Autismo (TEA), descritas pelo Ministério da Saúde (Brasil, 2014).

Assim, este estudo objetiva demonstrar o desenvolvimento de um aplicativo sobre detecção precoce do autismo à luz das Diretrizes para Atenção à Reabilitação do Transtorno do Espectro Autista (TEA), descritas pelo Ministério da Saúde, para dar subsídios ao ensino de profissionais de saúde e suas práticas educativas.

\section{MÉTODOS}

Este estudo apresenta uma abordagem qualitativa, do tipo descritiva exploratória. O aplicativo refere-se ao produto de uma dissertação de mestrado. Foi desenvolvido após uma pesquisa sobre a avaliação de acadêmicos e residentes de fisioterapia e terapia ocupacional sobre detecção precoce do TEA. $O$ estudo foi primeiramente submetido ao Comitê de Ética em Pesquisa (CEP) da Universidade do estado do Pará (UEPA) e aprovado no dia 31 de maio de 2016, sob número CAAE 55471316.0.0000.5174. Durante este estudo 
identificou-se que residentes e acadêmicos de fisioterapia e terapia ocupacional, que participaram da pesquisa, não possuem os conhecimentos necessários para realizar a detecção precoce do TEA em programas de vigilância do desenvolvimento infantil.

Buscando suprir está carência na formação destes alunos, desenvolveu-se um aplicativo no qual os procedimentos metodológicos seguiram as seguintes etapas: revisão bibliográfica, estudo das Diretrizes de Atenção à Reabilitação da Pessoa com Transtornos do Espectro do Autismo (Brasil, 2014) e produção técnica do aplicativo.

No primeiro momento realizou-se uma pesquisa bibliográfica nas seguintes bases de dados: SCIELO, LILACS, MEDLINE e PUBMED. Utilizou-se para a busca a combinação simples de três descritores: aplicativo AND diagnóstico AND autismo. Esta busca objetivou identificar na literatura brasileira estudos sobre o uso de aplicativos como ferramenta de auxílio para o diagnóstico do TEA. Utilizou-se como critérios de inclusão: artigos publicados nos últimos 20 anos, textos completos, com temas relacionados ao contexto brasileiro e que abordassem o assunto sobre diagnóstico do TEA. Não foram encontrados nenhum artigo com esta temática em nenhuma base de dados.

Assim, no segundo momento realizou-se o estudo detalhado das Diretrizes de Atenção à Reabilitação da Pessoa com TEA (BRASIL, 2014). Priorizou-se este documento, por este ser o material teórico disponibilizado pelo Ministério da Saúde no Brasil, servindo como guia para todos os profissionais e estudantes que atuam com pessoas com diagnóstico de TEA em todos os contextos de atenção em saúde.

No terceiro momento, com o auxílio de um tecnólogo em redes de computadores e através da ferramenta App Inventor foi desenvolvido o aplicativo que poderá ser disponibilizado para smartfones e tablets com sistema operacional Android.

O App Inventor é uma ferramenta gratuita, disponibilizada na internet para a programação de aplicativos via WEB. Foi construída a partir da parceria entre o Google e o Instituto de Tecnologia de Massachusett (MIT), com o objetivo de desenvolver uma interface visual, que permita que qualquer pessoa programe aplicativos, mesmo sem saber construir linhas de código e compilar programas. Atualmente, esta ferramenta é administrada pela equipe do Centro de Aprendizagem Móvel do MIT, com a colaboração do Laboratório de Ciências da Computação e Inteligência Artificial (CSAIL) do mesmo Instituto de Ensino (INSTITUTO DE TECNOLOGIA DE MASSACHUSETTS, 2017).

\section{RESULTADOS}

Os resultados deste estudo referem-se a produção do aplicativo SINAIS como ferramenta de ensino e aprendizagem. Foram seguidas as seguintes etapas: planejamento, execução e apresentação do aplicativo.

No primeiro momento foi feito o planejamento para definir o conteúdo e os itens que seriam utilizados, para posterior formulação das perguntas, priorizando-se uma linguagem clara, objetiva e de fácil compressão para garantir o acesso a qualquer usuário. Todo o conteúdo utilizado no aplicativo está de acordo com as Diretrizes de Atenção à Reabilitação da Pessoa com Transtornos do Espectro do Autismo (TEA), elaboradas pelo Ministério da Saúde.

Neste documento todos os sinais de alerta para o TEA foram organizados por faixa etária de: zero a seis meses, seis a 12 meses, 12 a 18 meses, 18 a 24 meses e 24 a 36 meses. Para cada faixa etária foram descritos um conjunto comportamentos a seguir (Brasil, 2014).

Crianças de 0 a 6 meses podem não acompanhar/buscar o olhar do cuidador, prestar mais atenção aos objetos do que a pessoas, ignorar ou apresentar pouca resposta aos sons de fala, tender ao silêncio e/ou a gritos aleatórios, choro indistinto em diferentes ocasiões, ou crises frequentes de choro duradouro, sem ligação aparente a evento ou pessoa e ausência ou raridade de comportamentos exploratórios (BRASIL, 2014). 
Durante os 06 a 12 meses de idade pode-se observar ausência de comportamentos antecipatórios e imitativos, alteração na expressão facial, balbucio pouco diferenciado, baixa presença de sorrisos.

Durante as tentativas de trocas comunicativas do avaliador a criança pode não apresentar comportamentos com intenção comunicativa, não atender ao ser chamado pelo nome, não repetir gestos em resposta a uma solicitação. A criança também pode precisar de muita insistência do adulto para se engajar em brincadeiras (BRASIL, 2014).

Em crianças de 12 a 18 meses observa-se ausência ou raridade do apontar, prejuízo na atenção compartilhada, ausência ou baixa frequência na emissão de palavras, nem mesmo por repetição, pouca variação na expressão facial, pouca exploração de objetos, fixação em algumas partes de objetos sem explorar suas funções e dificuldade em jogos de "faz de conta" (BRASIL, 2014).

Crianças com 18 a 24 meses podem apresentar prejuízo na atenção compartilhada, ecolalia, ausência de gestos durante a comunicação com o cuidador, dificuldade em imitar ações de adultos, principalmente durante episódios de brincadeira BRASIL, 2014).

Em crianças de 24 a 36 meses pode-se observar dificuldade na capacidade de comentar e/ou fazer perguntas sobre objetos ou situações que são compartilhadas, prejuízo na atenção compartilhada, os gestos e comentários em resposta ao adulto aparecem isoladamente ou após muita insistência do cuidador, iniciativas de comunicação são raras. Podem apresentar ainda, dificuldade ou desinteresse em narrativas referentes ao cotidiano, dificuldades em brincar perto de outras crianças, ainda que não necessariamente com elas (BRASIL, 2014).

Assim diante destas informações foram formuladas as questões que iriam compor o aplicativo. Após o planejamento da estrutura do conteúdo, discutiu-se com o tecnólogo em processamento de dados o passo a passo para o desenvolvimento do aplicativo.

Para cada faixa etária descrita anteriormente foi disponibilizado um conjunto de 7 a 15 perguntas. Todas as perguntas estavam relacionadas aos fatores de risco para a ocorrência do TEA em crianças, a partir das orientações coletadas nas Diretrizes.

O aplicativo possui um total de 55 perguntas organizadas de acordo com as faixas etárias: 0-6 meses, 6 12 meses, $12-18$ meses, 18-24 meses, 24-36 meses. As perguntas são direcionadas aos pais ou cuidadores da criança, que deverão responder a cada pergunta apertando os botões de SIM ou NÃO apresentados no final de cada pergunta.

Optou-se também por uma interface gráfica simples, evitando com isso a distração do usuário durante o uso do aplicativo, contribuindo para um acesso rápido e fácil. Inicialmente o usuário do aplicativo é direcionado a tela inicial, esta primeira tela apresenta o nome, a logomarca e um botão de PRÓXIMO, que possui a função de conduzir os usuários as telas secundárias (Figura 1).

O aplicativo recebeu o nome de SINAIS, para retratar aos usuários o objetivo a ser alcançado através do seu uso, ou seja, identificar os sinais de alerta para o TEA. Em um segundo momento, realizou-se o desenvolvimento da logomarca com o suporte de um profissional de multimídia.

Utilizou-se uma logomarca com formato circular, uma referência ao desenvolvimento humano. Optou-se por tons variados de azul, pois historicamente esta cor é utilizada durante as ações desenvolvidas com crianças com TEA. A logomarca apresenta mais de um tom de azul para ilustrar o espectro do transtorno, como uma condição que pode apresentar muitas variações quanto a sua manifestação clínica. Por fim, os tons de azul foram organizados a partir da ilustração de peças de um quebra-cabeça, uma alusão à necessidade de se identificar várias características e sintomas, que em conjunto possibilitarão a detecção precoce.

Ao utilizar o botão PRÓXIMO, o usuário terá acesso a segunda e a terceira tela. Cada tela irá fornecer as instruções iniciais quanto ao uso da ferramenta. (Figura 2). 
Figura 1 - Visão geral da página inicial do aplicativo SINAIS.

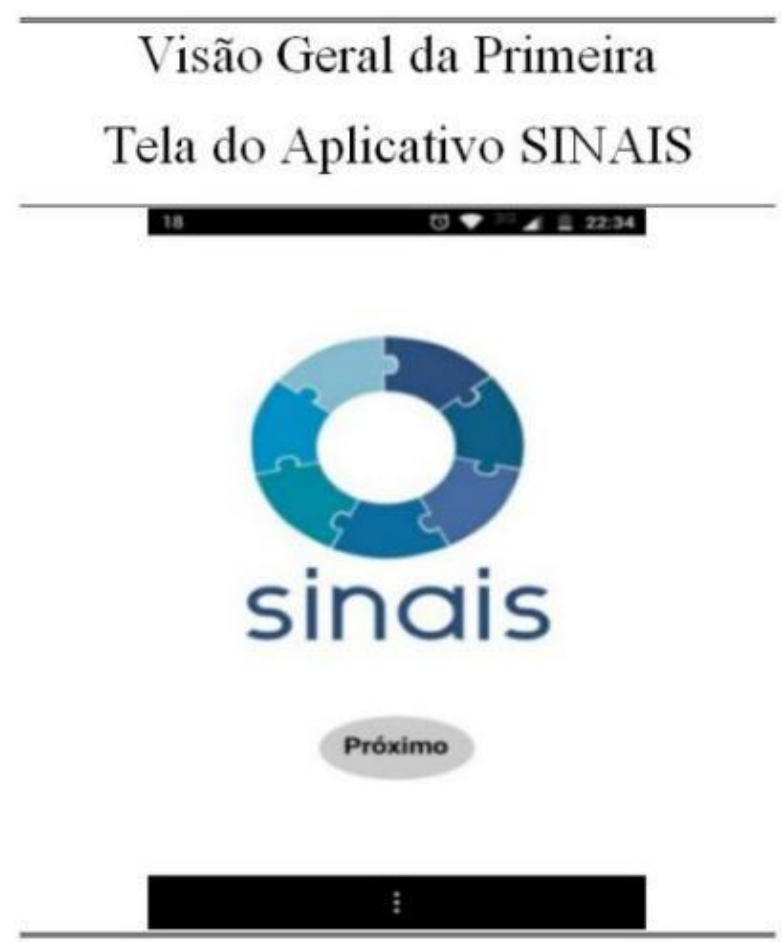

Fonte: Dados da pesquisa, 2017.

Figura 2 - Visão geral da segunda e da terceira tela do aplicativo SINAIS.

\begin{tabular}{ccc}
\hline Segunda e Terceira tela do Aplicativo SINAIS & Terceira tela \\
\hline $\begin{array}{c}\text { Segunda tela } \\
\text { resporguntas a seguir devem ser } \\
\text { com a presença de alguém que } \\
\text { convive com a criança e um } \\
\text { acadêmico ou profissional de } \\
\text { saúde. }\end{array}$ & $\begin{array}{c}\text { RESPONDA AS PERGUNTAS A } \\
\text { SEGUIR COM SIM OU NÃO. }\end{array}$ \\
& $\begin{array}{c}\text { Se um comportamento não for } \\
\text { observado com frequência, } \\
\text { ou ocorrer eventualmente, } \\
\text { responder com não. }\end{array}$ \\
\hline Próximo & Começar \\
\hline
\end{tabular}

Fonte: Dados da pesquisa, 2017. 
$\mathrm{Na}$ segunda tela receberá a orientação de que as perguntas do teste devem ser respondidas preferencialmente por alguém que convive com a criança, para garantir a fidedignidade das respostas,. É sugerido também a presença de um acadêmico ou profissional de saúde, para garantir a oferta de orientações adequadas.

Na terceira tela o usuário é orientado a responder a cada pergunta através dos botões de SIM ou NÃO. Caso a pergunta esteja relacionada a um comportamento da criança não observado com frequência, ou que ocorre eventualmente, o usuário terá assim que responder a esta pergunta acionando o botão NÃO.

Após estas orientações o usuário poderá iniciar o teste selecionando o botão COMEÇAR. Após este acionamento o usuário terá acesso à quarta tela do aplicativo, que contém o menu com as faixas etárias a serem escolhidas (Figura 3).

Figura 3 - Visão geral da quarta tela do aplicativo SINAIS.

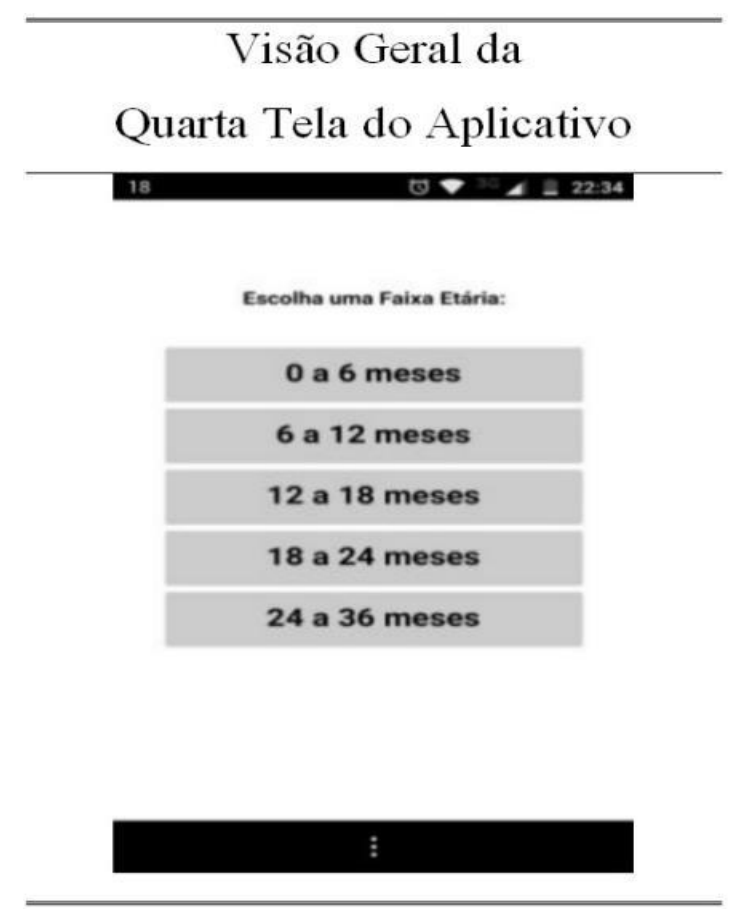

Fonte: Dados da pesquisa, 2017.

Quadro 1 - Perguntas do aplicativo SINAIS na faixa etária de 0 a 6 meses de idade.

\begin{tabular}{|c|}
\hline PERGUNTAS \\
\hline A criança acompanha e busca o olhar do cuidador? \\
\hline A criança presta mais atenção em pessoas do que em objetos? \\
\hline A criança presta atenção à voz, fala humana? \\
\hline A criança apresenta balbucio na presença ou ausência do cuidador? \\
\hline A criança apresenta vários tipos de choro dependendo da sua necessidade? \\
\hline A criança olha para objetos e os explora de várias formas? \\
\hline
\end{tabular}

Fonte: Dados da pesquisa, 2017. 
Utilizaram-se as mesmas faixas etárias descritas nas Diretrizes de Atenção à Reabilitação da Pessoa com TEA. A seguir será apresentado exemplos de perguntas utilizadas no aplicativo, que estão de acordo com a faixa etária de 0 a 6 meses de idade (Quadro 1).

Quando o usuário escolhe uma faixa etária, ele deverá responder a uma pergunta de cada vez, não havendo a possibilidade de pular perguntas. Após cada resposta o usuário é direcionado à pergunta seguinte, ao final da última pergunta, o teste será finalizado e o usuário será conduzido a próxima tela que irá indicar o percentual dos fatores risco.

Ressalta-se que a possibilidade de risco para o desenvolvimento do TEA estará relacionada com a ausência de características típicas do desenvolvimento infantil (BRASIL, 2014).

O fato da criança apresentar sinais de alerta para o TEA, sinais estes descritos pelas Diretrizes do Ministério da Saúde, não quer dizer que esta criança receberá o diagnóstico do transtorno.

A equipe que estiver atuando no programa de vigilância do desenvolvimento, no âmbito da atenção primária, deverá decidir sobre os encaminhamentos e procedimentos a serem realizados em função da especificidade de cada caso, e da disponibilidade do serviço na localidade (BRASIL, 2014). A seguir observase um exemplo de uma pergunta e de uma possível resultado do teste (Figura 4).

Figura 4 - Exemplos de telas referentes à pergunta e resultado do aplicativo SINAIS

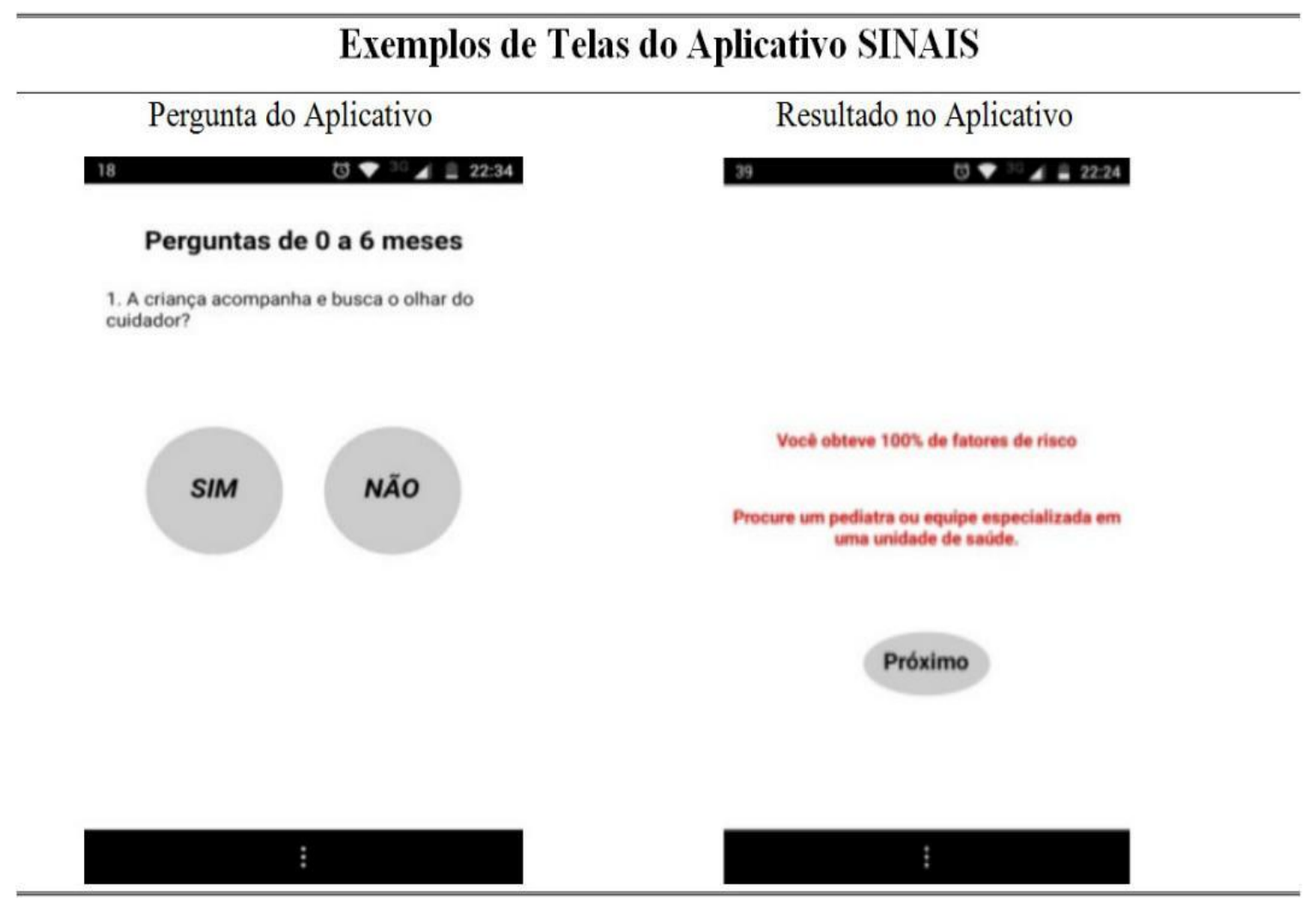

Fonte: Dados da pesquisa, 2017.

Destaca-se que o resultado gerado pelo aplicativo permitirá uma análise individual de cada criança, e que o seu uso não exclui a necessidade de se realizar outras avaliações de rastreio. O próprio aplicativo orienta o usuário a procurar um pediatra ou equipe especializada, em uma unidade de saúde, para que possa receber mais informações e seguir com os encaminhados caso seja necessário. 
Após o resultado, o usuário deverá seguir para a última tela acionando o botão de "PRÓXIMO", na qual terá acesso as informações da equipe técnica que desenvolveu o aplicativo e seus objetivos. Após a leitura, o usuário poderá escolher entre duas opções, refazer o teste ou sair do aplicativo.

Após a finalização do aplicativo o mesmo foi encaminhado ao Instituto Nacional da propriedade Industrial (INPE) para o registro de programas de computador sob o processo n. BR 512018 000167-0. O certificado de registro de programas de computador foi expedido no dia 14 de fevereiro de 2018.

\section{DISCUSSÃO}

Esta ferramenta foi desenvolvida para servir como um instrumento acessível de rastreio dos sinais de alerta para o TEA. E deve ser utilizado com o auxílio de um acadêmico ou profissional de saúde. Os estudos de Flores e Smeha (2013) e Muller (2012) destacam a necessidade do desenvolvimento de estratégias que auxiliem na identificação dos sinais de alerta para o TEA, pois identificaram que pediatras, neuropediatras e estudantes de medicina, também apresentam dificuldades em realizar a detecção precoce do TEA.

É importante frisar que, os instrumentos de rastreio são utilizados por profissionais de diversas áreas, alcançando assim uma maior abrangência, e objetivam apenas a detecção de sinais relativos que podem estar relacionados com o transtorno, ou seja, não determinam o diagnóstico (BRASIl, 2014). Em contrapartida, a realização de avaliações de rastreio com pais e cuidadores favorece que as informações colhidas apresentem maior acurácia, e assim a garantia de que as providências que serão tomadas a posteriori estarão de acordo com as reais necessidades da criança (ALCKMIN-CARVALHO et al, 2014).

Ressalta-se que não foi objetivo deste aplicativo substituir a atuação de uma equipe especializada, muito menos fornecer um diagnóstico definitivo para o TEA. Alckmin-Carvalho et al. (2014) reforça em seus estudos que as entrevistas com pais e cuidadores não podem substituir a avaliação de um profissional. E que um diagnóstico definitivo não deve ser feito utilizando apenas um único instrumento de rastreio.

O Ministério da Saúde, a partir das orientações descritas nas Diretrizes de Atenção à Reabilitação da Pessoa com Transtornos do Espectro do Autismo (TEA), recomenda que os instrumentos de rastreio sejam utilizados para fornecer informações sobre a detecção de sinais de alerta. Para a realização do diagnóstico definitivo a criança deverá ser encaminhada para um profissional capacitado (BRASIL, 2014).

Observa-se que, atualmente, o uso crescente de novas tecnologias na área da saúde contribui para aproximar os profissionais de saúde as novas estratégias de avaliação e diagnóstico, além de contribuir para o acesso e processamento rápido das informações. O uso de aplicativos móveis possibilita um diagnóstico rápido, intervindo diretamente na tomada de decisão, acompanhamento clínico e prognóstico (DOS SANTOS et al., 2017). Para Castro et al. (2015) é fundamental a elaboração de sistemas eficazes, que atendam as demandas do usuário do SUS de maneira ágil e que resulte em uma experiência positiva.

As ferramentas tecnológicas são uma nova possibilidade, atuando como instrumento de apoio educacional no processo de ensino e aprendizagem de acadêmicos da área da saúde, contribuindo para o bom desenvolvimento profissional, pois irá facilitar no processo de tomada de decisões clínicas, o aperfeiçoamento de conhecimentos e habilidades, tornando o aluno sempre atualizado com as necessidades de sua clientela, e assim, oferta subsídios para as práticas educativas realizadas em conjunto com a comunidade (WARMLING et al., 2012). O uso de aplicativos móveis garante ao processo de investigação diagnóstica uma análise e acesso rápido as informações coletadas, além de possibilitar a exibição de um resultado final mais conciso ( DOS SANTOS et al., 2017).

É necessário o desenvolvimento de mais estudos que tratem sobre dispositivos tecnológicos e de sua incorporação no SUS, pois as novas tecnologias atuam como instrumentos de apoio para o diagnóstico e tratamento de usuários, sendo fundamental identificar a relação de custo-efetividade para sua aplicação (CONTÓ et al., 2015). Contudo, é fundamental que as Instituições de Ensino Superior (IES) e seus docentes utilizem em suas práticas de trabalho o uso de tecnologias, como um mecanismo de produção de conhecimento, com impacto nas ações educativas e no atendimento à comunidade (WARMLING et al., 2012). 
Espera-se que o aplicativo SINAIS contribua para o meio científico como uma ferramenta de ensino que ajuda no rastreio de sinais de alerta do TEA, durante práticas educativas em saúde, com foco na prevenção, conforme é preconizado nas Diretrizes do SUS e políticas públicas em saúde no Brasil.

\section{CONCLUSÃO}

No contexto da crise entre o grande número de usuários do SUS e a oferta de serviços na atenção integral ao cuidado na infância, é fundamental o estabelecimento de ferramentas que busquem favorecer um atendimento mais rápido e resolutivo. Assim, o desenvolvimento do aplicativo SINAIS possibilitou vislumbrar sua aplicação em diversos contextos na atenção primária, principalmente em programas de vigilância do desenvolvimento. Espera-se que em pesquisas futuras seja possível identificar os benefícios de seu uso para a detecção precoce do TEA. Quanto ao ensino, espera-se que este trabalho favoreça a reflexão sobre a necessidade de um processo de ensino e aprendizagem inovador, com destaque para um aprendizado significativo, com participação ativa do aluno, a partir de uma análise crítica e reflexiva, com responsabilidade, baseada na tomada de decisões e da resolução de problemas a partir da aprendizagem móvel.

\section{AGRADECIMENTOS E FINANCIAMENTO}

Agradecemos a Universidade do Estado do Pará na qual foi realizado o mestrado e a Gabriela Vicari Flores, profissional de multimídia que contribui com a produção da logomarca do aplicativo SINAIS. Este estudo foi realizado com financiamento próprio.

\section{REFERÊNCIAS}

1. ALCKMIN-CARVALHO F, Teixeira MCTV, BRUNONI D et al. Identificação de sinais precoces de autismo segundo um protocolo de observação estruturada: um estudo de seguimento. Revista Psico, 2014; 45(4): 502-512.

2. ALCKMIN-CARVALHO F, DE PAULA CS, TEIXEIRA MCTV et al. Rastreamento de sinais precoces de transtorno do espectro do autismo em crianças de creches de um município de São Paulo. Revista Psicologia: Teoria e Prática, 2013;15(2): 144-154.

3. BARBA PCSD, SILVA RF, JOAQUIM RHVT et al. Formação inovadora em terapia ocupacional. Interface: comunicação Saúde Educação, 2012; 16(42): 829-842.

4. BARROS VFA, PINTO JR JS, BORGES RC et al. Aplicativo Móvel para Automação e Monitoração do Sistema de Atenção Primária a Saúde.Cadernos de Informática, 2011; 6(1): 241-244.

5. BRASIL. Ministério da Saúde. Secretaria de Atenção à Saúde. Departamento de Ações Programáticas Estratégicas. Diretrizes de Atenção à Reabilitação da Pessoa com Transtornos do Espectro do Autismo (TEA). Brasília, DF: Ministério da Saúde, 2014.

6. CAMPOS FE, BRENELLI SL, LOBO LC et al. O SUS como escola: a responsabilidade social com a atenção à saúde da população e com a aprendizagem dos futuros profissionais de saúde. Revista Brasileira de Educação Médica, 2009; 33(4): 513-514.

7. CASTRO E, REIS E, SPINILLO CG et al. Interface gráfico-informacional de aplicativos de educação em saúde: uma análise do app - Saúde da Criança I da UMA- SUS/UFMA. In: CONGRESSO internacional de desing da informação, 7. 2015, São Paulo. Anais...São Paulo, 2015.

8. CONTÓ M, BIELLA CA, PETRAMALE CA. Dispositivos médicos no Sistema Único de Saúde (SUS): avaliação e incorporação tecnológica . Revista Eletrônica Gestão \& Saúde, 2015; 6(4): 3016-3023.

9. DE OLIVEIRA ARF, ALENCAR MSM. O uso de aplicativos de saúde para dispositivos móveis com fontes de informação e educação em saúde. Revista Digital Biblioteconomia e Ciência da Informação, 2017; 15(1): 234-245.

10. DOS SANTOS TS, ALVES TB, FILHO FSY et al. Desenvolvimento de aplicativo para dispositivos móveis voltados para identificação do fenótipo de fragilidade em idosos. Revista Brasileira de Geriatria e Gerontologia, 2017; 20(1): 70-76.

11. FLORES MR, SMEHA LN. Bebês com risco de autismo: o não-olhar do médico. Ágora, 2013; 16(spe): 141-157.

12. INSTITUTO DE TECNOLOGIA DE MASSACHUSETTS. App Inventor. Disponível em: http://appinventor.mit.edu/explore/aboutus.html. Acesso em: 21 abr. 2017.

13. MULLER C. Conhecimento dos estudantes de medicina acerca do autismo em uma Universidade do Rio Grande do Sul, RS.Dissertação (Mestrado em Saúde da Criança e do Adolescente) - Faculdade de Medicina. Universidade Federal do Rio Grande do Sul, Porto Alegre, 2012; 73p.

14. NYGREN G, CEDERLUND M, SANDBERG E et al. The prevalence of autismo spectrum disorders intoddlers: a population study of 2-year-old swedish children. Journal Autism Desenvolviment Disorders, 2011; 42(7):1491-1497.

15. OZONOFF S, LOSIF AM, BAGUIO $\mathrm{F}$ et al. A prospective study of the emergence of early behavioral signs of autism. Journal of the American Academy of Child and Adolescent sychiatry, 2010; 49(3): 256-266.

16. SCHWARTZMAN JS, ARAÚJO C. A. Transtorno do Espectro do autismo. São Paulo: Memmon, 2011; $327 \mathrm{p}$.

17. TEMPSK P, BORBA M. O SUS como escola. Revista Brasileira de Educação Médica, 2009; 33(3): 319 -320.

18. WARMLING AMF, AMANTE CJ, VIEIRA MLH et al. Aplicativo baseado nos determinantes da doença cárie para apoio à tomada de decisão. Jornal Brasileiro TeleSSaúde, 2012; 1(2):35-42. 